\title{
Some Geometric Properties of Multivalent Convex Function for Operator on Hilbert Space
}

\author{
Mohammed Hadi Lafta ${ }^{a}$ \\ Mazin Hashim Suhhiem ${ }^{\mathrm{b}}$ \\ ${ }^{a}$ Department of Business Administration,college Administration and Economics, University of \\ Sumer,Dhi Qar, Iraq \\ ${ }^{b}$ Department of Statistical, college Administration and Economics, University of Sumer, Dhi Qar,Iraq \\ mmohamnedhadi@gmail.com_mazin.suhhiem@yahoo.com
}

Submission date:- 22/11/2017 Acceptance date:- 6/3/2018 Publication date:- 2/9/2018

Keywords: Multivalent function, Hilbert space, Distortion theorem, Radius of close-to- convexity, Extreme points, Closure theorem, Weighted mean.

\begin{abstract}
By making use of the operator on Hilbert space, we introduce and study some properties of geometric of

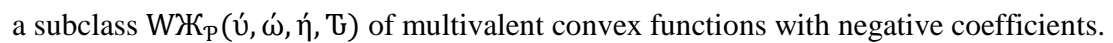

Also we obtain some geometric properties, such as, coefficient inequality, growth and distortion theorem, extreme points, convex set, closure theorem, radius of close-to-convexity, weighted mean and inclusive properties.
\end{abstract}

\section{Introduction}

In this paper, the aim is to mention the basic facts about geometric function theory, which this is obtained from mixing of geometry and analysis. Its origin started from the 19th century, but it continued and continually applicable till now. Geometric Function Theory is an important branch of complex analysis; It deals with the geometric properties of the analytic functions. In particular, we will concentrate on the important ideas in this theory. The fundamentals of this theory are explained in most text books on this subject. Also, we review and consider the basic ideas, principles, definitions and the general principles of complex analysis, which underline the geometric function theory of a complex variable rather than the basic lemmas which are needed in the proofs of our results. A full discussion of these principles can be found in standard text books, [1], [2].

The study of multivalent functions is one of the main branches of geometric function theory and plays a central role in complex analysis.

Let $W_{P}$ be the class of functions $f$ of the form:

$$
f(z)=z^{P}+\sum_{\tilde{n}=1}^{\infty} a_{\tilde{n}+P} z^{\tilde{n}+P}(P \in \mathbb{N}=\{1,2, \ldots\}),
$$

which are analytic and $P$ - valent in the open unit disk $U=\{z \in \mathbb{C}:|z|<1\}$. Let $\aleph_{P}$ denote the subclass of $W_{P}$ consisting of functions of the form:

$$
f(z)=z^{P}-\sum_{\tilde{n}=1}^{\infty} a_{\tilde{n}+P} z^{\tilde{n}+P}\left(a_{\tilde{n}+P} \geq 0, P \in \mathbb{N}=\{1,2, \ldots\}\right),
$$

Definition (1.1): A function $f \in W_{P}$ is said to be in the class $W \aleph_{P}\left(v^{\prime}, \omega^{\prime}, \eta ́\right)$ if it satisfies

$$
\left|\frac{z f^{\prime \prime}(z)+2 f^{\prime}(z)-P(1+P) z^{P-1}}{\hat{v}\left(z f^{\prime \prime}(z)+2 f^{\prime}(z)-P(\dot{\omega})+P(P-\dot{\omega}+1)\right.}\right|<0,
$$


where $0 \leq \dot{v}<1,0 \leq \dot{\omega}<\mathrm{P}, 0<\eta^{\prime} \leq 1$ dan $z \in U^{\prime}$.

Let $\mathrm{H}$ be a Hilbert space on the complex field. Let $\mathrm{G}$ be a linear operator on $\mathrm{H}$. For a complex analytic function $f$ on the unit disk $U$, we denoted $f(\mathrm{~T})$, the operator on $\mathrm{H}$ defined by the usual Riesz- Dunford integral [3]

$$
f(\mathrm{G})=\frac{1}{2 \pi \mathrm{i}} \int_{\mathcal{C}} f(z)(z I-\mathrm{T})^{-1} \mathrm{dz},
$$

where I is the identity operator on $\mathrm{H}, \boldsymbol{C}$ is a positively oriented simple closed rectifiable contour lying in $\mathrm{U}^{3}$ and containing the spectrum $\kappa(\mathrm{G})$ of $\mathrm{G}$ in its interiOr domain [4]. Also $f(\mathrm{G})$ can be defined by the series

$$
f(\mathrm{~T})=\sum_{\tilde{\mathrm{n}}=0}^{\infty} \frac{f^{(\tilde{\mathrm{n}})}(0)}{\tilde{\mathrm{n}} !} \mathrm{T}^{\tilde{\mathrm{n}}}
$$

which converges in the norm topology [5].

Definition (1.2): Let $\mathrm{H}$ be a Hilbert space and $\mathrm{T}$ be an operator on $\mathrm{H}$ such that $\mathrm{T} \neq \varphi$

and $\|\mathrm{T}\|<1$. Let $v$, $\omega$ be real numbers such that $0 \leq \dot{v}<1,0 \leq \dot{\omega}<\mathrm{P}, 0<\dot{\eta} \leq 1$.

An analytic function $f$ on the unit disk belong to the class $W \aleph_{\mathrm{P}}\left(v^{\prime}, \omega^{\prime}, \eta^{\prime}, \mathrm{G}\right)$ if it satisfy the inequality

$\left\|\mathrm{G} f^{\prime \prime}(\mathrm{T})+2 f^{\prime}(\mathrm{G})-\mathrm{P}(1+\mathrm{P}) \mathrm{G}^{\mathrm{P}-1}\right\|<\eta^{\prime}\left\|\dot{v}\left(\mathrm{G} f^{\prime \prime}(\mathrm{G})+2 f^{\prime}(\mathrm{G})-\mathrm{P} \dot{\omega}\right)+\mathrm{P}(\mathrm{P}-\dot{\omega}+1)\right\|$,

The operator on Hilbert space were consider recently by [6]-[12].

\section{Main results:}

The first theorem gives Coefficient inequality for a function $f$ to be in the class $W \aleph_{\mathrm{P}}\left(\dot{v}^{\prime}, \hat{\omega}, \eta \dot{\eta}, \mathrm{T}\right)$.

Theorem (2.1): Let $f \in \aleph_{\mathrm{P}}$ be defined by (2). Then $f \in W \aleph_{\mathrm{P}}\left(\hat{v}^{\prime}, \omega^{\prime}, \eta^{\prime}, \mathrm{T}\right)$ for all $\mathrm{G} \neq \varphi$ if and only if

$$
\sum_{\tilde{n}=1}^{\infty}(\tilde{\mathrm{n}}+\mathrm{P})(\tilde{\mathrm{n}}+\mathrm{P}+1)(1+\eta \dot{\eta}) a_{\tilde{\mathrm{n}}+\mathrm{P}} \leq \tilde{\eta} \mathrm{P}(\dot{v}+1)(\mathrm{P}-\dot{\omega}+1) \text {. }
$$

where $0 \leq \dot{v}<1,0 \leq \dot{\omega}<\mathrm{P}, 0<\dot{\eta} \leq 1$.

The result is sharp for the function $f$ given by

$$
f(z)=z^{P}-\frac{\dot{\eta}^{P}(\dot{u}+1)(P-\dot{\omega}+1)}{(\tilde{n}+P)(\tilde{n}+P+1)\left(1+\eta^{\prime}\right)} z^{\tilde{n}+P}, \tilde{n} \geq 1 .
$$

Proof: Suppose that the inequality (3) holds. Then, we have

$$
\begin{aligned}
& \left\|\mathrm{T} f^{\prime \prime}(\mathrm{G})+2 f^{\prime}(\mathrm{G})-\mathrm{P}(1+\mathrm{P}) \mathrm{G}^{\mathrm{P}-1}\right\|-\eta^{\prime}\left\|v^{\prime}\left(\mathrm{G} f^{\prime \prime}(\mathrm{G})+2 f^{\prime}(\mathrm{G})-\mathrm{P} \omega\right)+\mathrm{P}(\mathrm{P}-\dot{\omega}+1)\right\| \\
& \left\|-\sum_{\tilde{n}=1}^{\infty}(\tilde{n}+P)(\tilde{n}+P+1) a_{\tilde{n}+P} T^{\tilde{n}+P-1}\right\| \\
& -\eta^{\prime}\left\|\dot{v} \mathrm{P}(\mathrm{P}+1) \mathrm{G}^{\mathrm{P}-1}-\sum_{\tilde{\mathrm{n}}=1}^{\infty} u^{\prime}(\tilde{\mathrm{n}}+\mathrm{P})(\tilde{\mathrm{n}}+\mathrm{P}+1) a_{\tilde{n}+\mathrm{P}} \mathrm{T}^{\tilde{n}+\mathrm{P}-1}-u^{\prime} \mathrm{P} \dot{\omega}+\mathrm{P}(\mathrm{P}-\dot{\omega}+1)\right\|
\end{aligned}
$$

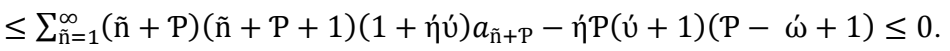

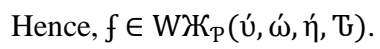

To show the converse, let $f \in W \aleph_{\mathrm{P}}\left(\dot{v}^{\prime}, \omega^{\prime}, \eta \dot{\eta}, \mathrm{U}\right)$. Then

$\left\|\mathrm{G} f^{\prime \prime}(\mathrm{G})+2 f^{\prime}(\mathrm{G})-\mathrm{P}(1+\mathrm{P}) \mathrm{G}^{\mathrm{P}-1}\right\|<\eta^{\prime}\left\|\dot{u}\left(\mathrm{G} f^{\prime \prime}(\mathrm{G})+2 f^{\prime}(\mathrm{T})-\mathrm{P} \omega\right)+\mathrm{P}(\mathrm{P}-\dot{\omega}+1)\right\|$, gives

$\left\|-\sum_{\tilde{n}=1}^{\infty}(\tilde{n}+P)(\tilde{n}+P+1) a_{\tilde{n}+P} G^{\tilde{n}+P-1}\right\|$ 
$<\eta^{\prime}\left\|\hat{v} \mathrm{P}(\mathrm{P}+1) \mathrm{T}^{\mathrm{P}-1}-\sum_{\tilde{\mathrm{n}}=1}^{\infty} \dot{v}(\tilde{\mathrm{n}}+\mathrm{P})(\tilde{\mathrm{n}}+\mathrm{P}+1) a_{\tilde{\mathrm{n}}+\mathrm{P}} \mathrm{G}^{\tilde{n}+\mathrm{P}-1}-\mathrm{u}^{\mathrm{P}} \dot{\omega}+\mathrm{P}(\mathrm{P}-\dot{\omega}+1)\right\|$.

Setting $\mathrm{T}=x I(0<x<1)$ in the above inequality, we get

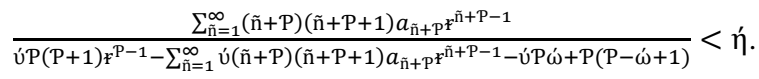

Up0n clearing denominator in (5) and letting $r \rightarrow 1$, we obtain

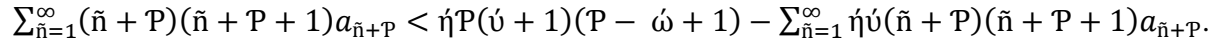

Thus

$$
\sum_{\tilde{n}=1}^{\infty}(\tilde{n}+P)(\tilde{n}+P+1)(1+\eta ́ v ́) a_{\tilde{n}+P} \leq \eta^{P} P(\dot{v}+1)(P-\dot{\omega}+1)
$$

which completes the proof.

Corollary (2.1): If $f \in W \aleph_{P}\left(v^{\prime}, \dot{\omega}, \eta \dot{\eta}, \mathrm{T}\right)$, then

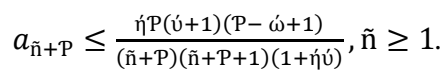

Next, we obtain the growth and distortion for a function $f$ to be in the class $W \aleph_{\mathrm{p}}\left(\hat{v}^{\prime}, \dot{\omega}, \eta \dot{\eta}, \mathrm{T}\right)$.

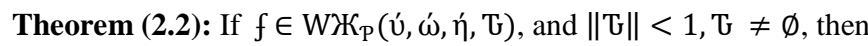

$$
\|\mathrm{T}\|^{\mathrm{P}}-\frac{\eta^{\prime} \mathrm{P}(\dot{v}+1)(\mathrm{P}-\dot{\omega}+1)}{(\mathrm{P}+1)(\mathrm{P}+2)(1+\eta \dot{u})}\|\mathrm{T}\|^{\mathrm{P}+1} \leq\|f(\mathrm{~T})\| \leq\|\mathrm{T}\|^{\mathrm{P}}+\frac{\eta^{\mathrm{P}} \mathrm{P}(\dot{v}+1)(\mathrm{P}-\dot{\omega}+1)}{(\mathrm{P}+1)(\mathrm{P}+2)(1+\eta \dot{u})}\|\mathrm{T}\|^{\mathrm{P}+1}
$$

and

$$
\mathrm{P}\|\mathrm{G}\|^{\mathrm{P}-1}-\frac{\eta^{\mathrm{P}}(\dot{v}+1)(\mathrm{P}-\dot{\omega}+1)}{(\mathrm{P}+2)\left(1+\eta^{\prime}\right)}\|\mathrm{T}\|^{\mathrm{P}} \leq\left\|f^{\prime}(\mathrm{T})\right\| \leq \mathrm{P}\|\mathrm{T}\|^{\mathrm{P}-1}-\frac{\eta^{\mathrm{P}} \mathrm{P}(\dot{v}+1)(\mathrm{P}-\dot{\omega}+1)}{(\mathrm{P}+2)\left(1+\eta^{\prime}\right)}\|\mathrm{G}\|^{\mathrm{P}} .
$$

The result is sharp for the function $f$ given by

$$
f(z)=z^{P}-\frac{\eta^{P}(\dot{v}+1)(P-\dot{\omega}+1)}{(P+1)(P+2)\left(1+\dot{\eta}^{\prime}\right)} z^{P+1} .
$$

Proof: According to the Theorem (2.1), we get

$$
\sum_{\tilde{\mathrm{n}}=1}^{\infty} a_{\tilde{\mathrm{n}}+\mathrm{P}} \leq \frac{\eta \dot{\mathrm{P}} \mathrm{P}(\dot{v}+1)(\mathrm{P}-\dot{\omega}+1)}{(\mathrm{P}+1)(\mathrm{P}+2)(1+\eta \dot{u})} .
$$

Hence

$$
\begin{aligned}
\|f(\mathrm{G})\| \geq\|\mathrm{G}\|^{\mathrm{P}}-\sum_{\tilde{\mathrm{n}}=1}^{\infty} a_{\tilde{\mathrm{n}}+\mathrm{P}}\|\mathrm{T}\|^{\tilde{\mathrm{n}}+\mathrm{P}} \\
\quad \geq\|\mathrm{T}\|^{\mathrm{P}}-\|\mathrm{G}\|^{\mathrm{P}+1} \sum_{\tilde{n}=1}^{\infty} a_{\tilde{\mathrm{n}}+\mathrm{P}} \\
\geq\|\mathrm{G}\|^{\mathrm{P}}-\frac{\dot{\eta}^{\mathrm{P}(\dot{v}+1)(\mathrm{P}-\dot{\omega}+1)}}{(\mathrm{P}+1)(\mathrm{P}+2)(1+\eta \dot{u})}\|\mathrm{G}\|^{\mathrm{P}+1} .
\end{aligned}
$$

Also,

$$
\begin{aligned}
\|f(\mathrm{G})\| & \leq\|\mathrm{G}\|^{\mathrm{P}}+\sum_{\tilde{n}=1}^{\infty} a_{\tilde{\mathrm{n}}+\mathrm{P}}\|\mathrm{G}\|^{\tilde{\mathrm{n}}+\mathrm{P}} \\
& \leq\|\mathrm{G}\|^{\mathrm{P}}+\frac{\eta^{\mathrm{P}} \mathrm{P}(\dot{v}+1)(\mathrm{P}-\dot{\omega}+1)}{(\mathrm{P}+1)(\mathrm{P}+2)(1+\dot{\eta} u)}\|\mathrm{T}\|^{\mathrm{P}+1} .
\end{aligned}
$$

In view of (Theorem 2.1), we have 


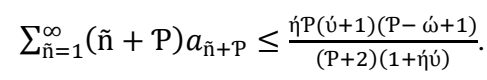

Thus

$$
\begin{aligned}
\left\|f^{\prime}(\mathrm{T})\right\| & \geq \mathrm{P}\|\mathrm{T}\|^{\mathrm{P}-1}-\sum_{\tilde{\mathrm{n}}=1}^{\infty}(\tilde{\mathrm{n}}+\mathrm{P}) a_{\tilde{\mathrm{n}}+\mathrm{P}}\|\mathrm{T}\|^{\tilde{\mathrm{n}}+\mathrm{P}-1} \\
& \geq \mathrm{P}\|\mathrm{T}\|^{\mathrm{P}-1}-\|\mathrm{T}\|^{\mathrm{P}} \sum_{\tilde{\mathrm{n}}=1}^{\infty}(\tilde{\mathrm{n}}+\mathrm{P}) a_{\tilde{\mathrm{n}}+\mathrm{P}} \\
& \geq \mathrm{P}\|\mathrm{T}\|^{\mathrm{P}-1}-\frac{\eta^{\mathrm{P}(\dot{v}+1)(\mathrm{P}-\dot{\omega}+1)}}{(\mathrm{P}+2)(1+\eta \dot{v})}\|\mathrm{T}\|^{\mathrm{P}}
\end{aligned}
$$

and

$$
\begin{aligned}
\left\|f^{\prime}(\mathrm{G})\right\| & \leq \mathrm{P}\|\mathrm{G}\|^{\mathrm{P}-1}-\|\mathrm{G}\|^{\mathrm{P}} \sum_{\tilde{\mathrm{n}}=1}^{\infty}(\tilde{\mathrm{n}}+\mathrm{P}) a_{\tilde{\mathrm{n}}+\mathrm{P}} \\
& \leq \mathrm{P}\|\mathrm{G}\|^{\mathrm{P}-1}-\frac{\eta^{\mathrm{P}}(\dot{\mathrm{v}}+1)(\mathrm{P}-\dot{\omega}+1)}{(\mathrm{P}+2)(1+\dot{\eta} \dot{v})}\|\mathrm{G}\|^{\mathrm{P}} .
\end{aligned}
$$

Therefore the proof is complete.

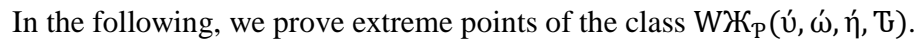

Theorem (2.3): Let $f_{0}(z)=z^{P}$ and

$$
f_{\tilde{n}}(z)=z^{P}-\frac{\eta^{\prime} P(\dot{v}+1)(P-\dot{\omega}+1)}{(\tilde{n}+P)(\tilde{n}+P+1)(1+\eta \dot{u})} z^{\tilde{n}+P}, \tilde{n} \geq 1
$$

Then $f \in W \aleph_{\mathrm{P}}\left(\mathcal{u}^{\prime}, \dot{\omega}, \dot{\eta}^{\prime}, \mathrm{U}\right)$ if and only if it can be expressed in the form

$$
f(z)=\sum_{\tilde{n}=0}^{\infty} B_{\tilde{n}} f_{\tilde{n}}(z)
$$

where $B_{\tilde{\mathrm{n}}} \geq 0$ and $\sum_{\tilde{\mathrm{n}}=0}^{\infty} B_{\tilde{\mathrm{n}}}=1$.

Proof: Assume that $f$ can be expressed by (6). Then, we have

$$
f(z)=\sum_{\tilde{n}=0}^{\infty} B_{\tilde{n}} f_{\tilde{n}}(z)=z^{P}-\sum_{\tilde{n}=0}^{\infty} \frac{\eta^{\prime} P(\dot{v}+1)(P-\dot{\omega}+1)}{(\tilde{n}+P)(\tilde{n}+P+1)(1+\eta \dot{u})} B_{\tilde{n}} z^{\tilde{n}+P} .
$$

Thus

$$
\sum_{\tilde{\mathrm{n}}=0}^{\infty} \frac{(\tilde{\mathrm{n}}+\mathrm{P})(\tilde{\mathrm{n}}+\mathrm{P}+1)(1+\dot{\eta} \dot{v})}{\eta^{\mathrm{P}} \mathrm{P}(\dot{v}+1)(\mathrm{P}-\dot{\omega}+1)} \frac{\dot{\eta} \mathrm{P}(\dot{v}+1)(\mathrm{P}-\dot{\omega}+1)}{(\tilde{\mathrm{n}}+\mathrm{P})(\tilde{\mathrm{n}}+\mathrm{P}+1)(1+\eta \dot{u})} B_{\tilde{\mathrm{n}}}=\sum_{\tilde{\mathrm{n}}=0}^{\infty} B_{\tilde{\mathrm{n}}}=1-B_{0} \leq 1,
$$

and so $f \in W \aleph_{\mathrm{P}}\left(v^{\prime}, \dot{\omega}, \eta \dot{\eta}, \mathrm{U}\right)$.

Conversely, Suppose that $f$ given by (2) in the class $W \aleph_{\mathrm{P}}\left(v^{\prime}, \omega^{\prime}, \eta^{\prime}, \mathrm{T}\right)$. Then by

Corollary (2.1), we have

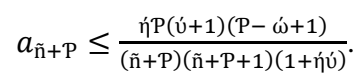

Setting

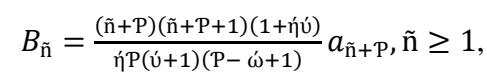

and $B_{\tilde{\mathrm{n}}}=1-\sum_{\tilde{\mathrm{n}}=1}^{\infty} B_{\tilde{\mathrm{n}}}$. Then

$$
f(z)=\sum_{\tilde{n}=0}^{\infty} B_{\tilde{n}} f_{\tilde{n}}(z),
$$


This completes the proof of the theorem.

Next, we obtain the convex set of the class $W \aleph_{\mathrm{P}}\left(v^{\prime}, \dot{\omega}, \eta^{\prime}, \mathrm{T}\right)$.

Theorem (2.4): The class $W \aleph_{\mathrm{P}}\left(v^{\prime}, \omega^{\prime}, \eta, \mathrm{T}\right)$ is a convex set.

Proof: Let $f_{1}$ and $f_{2}$ be the arbitrary element of $W \aleph_{\mathrm{P}}\left(\mathcal{v}^{\prime}, \dot{\omega}, \eta \dot{\eta}, \mathrm{U}\right)$. Then for every $¥(0 \leq \nsucceq \leq 1)$, we show that $(1-\nsucceq) f_{1}+\nsucceq f_{2} \in W \aleph_{P}\left(v^{\prime}, \dot{\omega}, \eta \dot{\eta}, \mathrm{T}\right)$. Thus, we have

$$
(1-\nsucceq) f_{1}+\nsucceq f_{2}=z^{P}-\sum_{\tilde{n}=1}^{\infty}\left((1-\nsucceq) a_{\tilde{n}+P}+\nsucceq b_{\tilde{n}+P}\right) z^{\tilde{n}+P} .
$$

Hence,

$$
\begin{aligned}
& \sum_{\tilde{n}=1}^{\infty}(\tilde{n}+P)(\tilde{n}+P+1)\left(1+\eta_{u}\right)\left((1-\nsucceq) a_{\tilde{n}+P}+\nsucceq b_{\tilde{n}+P}\right)
\end{aligned}
$$

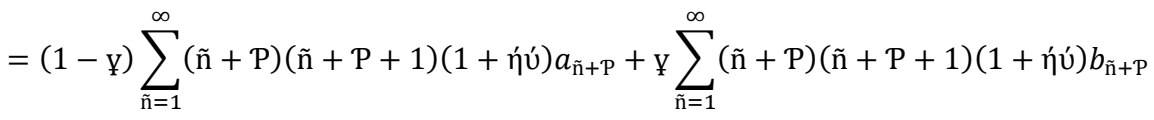

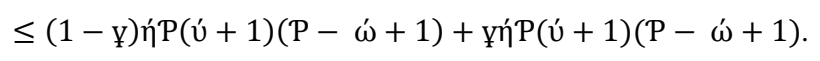

This completes the proof.

We will consider the function $f_{\hat{r}}(z)$ defined, for every $\hat{r}=1,2,3, \ldots, \dot{\varepsilon}$ by

$$
f_{\hat{r}}(z)=z^{P}-\sum_{\tilde{n}=1}^{\infty} a_{\tilde{n}+P, \hat{r}} z^{\tilde{n}+P}\left(a_{\tilde{n}+P, \hat{r}} \geq 0\right) .
$$

Next, we discuss the closure theorem.

Theorem (2.5): Let the function $f_{\hat{r}}(z)$ defined by (9) be in the class $W \aleph_{\mathrm{P}}(\dot{v}, \dot{\omega}, \dot{\eta}, \mathrm{T})$.

Then the function $G(z)$ defined by

$$
G(z)=\sum_{\hat{\mathrm{r}}=1}^{\grave{\varepsilon}} \hat{\mathrm{e}}_{\hat{\mathrm{r}}} f_{\hat{\mathrm{r}}}(z) \text { and } \sum_{\grave{\mathrm{r}}=1}^{\grave{\varepsilon}} \hat{\mathrm{e}}_{\hat{\mathrm{r}}}=1, \hat{\mathrm{e}}_{\hat{\mathrm{r}}} \geq 0
$$

is in the class $W \aleph_{P}(\dot{v}, \dot{\omega}, \eta \dot{\eta}, \mathrm{T})$.

Proof: By using definition of $G(z)$, we get

$$
G(z)=\left(\sum_{\hat{\mathrm{r}}=1}^{\grave{\varepsilon}} \hat{\mathrm{e}}_{\hat{\mathrm{r}}}\right) z^{\mathrm{P}}-\sum_{\tilde{\mathrm{n}}=1}^{\infty}\left(\sum_{\hat{\mathrm{r}}=1}^{\grave{\varepsilon}} \hat{\mathrm{e}}_{\hat{\mathrm{r}}} a_{\tilde{\mathrm{n}}+\mathrm{P}, \hat{\mathrm{r}}}\right) z^{\tilde{n}+P}
$$

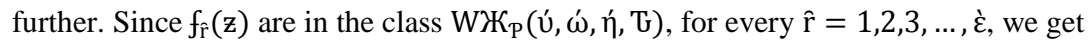

$$
\sum_{\tilde{n}=1}^{\infty}(\tilde{\mathrm{n}}+\mathrm{P})(\tilde{\mathrm{n}}+\mathrm{P}+1)\left(1+\eta^{\prime} u^{\prime}\right) a_{\tilde{\mathrm{n}}+\mathrm{P}, \hat{\mathrm{r}}} \leq \eta^{\prime} \mathrm{P}\left(\hat{u}^{\prime}+1\right)\left(\mathrm{P}-\omega^{\prime}+1\right)
$$

for every $\hat{r}=1,2,3, \ldots, \grave{\varepsilon}$.

Hence, we can see that

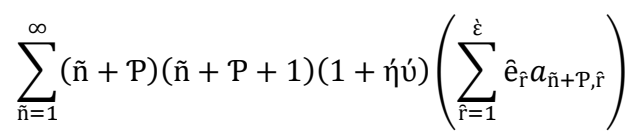

$=\sum_{\hat{\mathrm{r}}=1}^{\grave{\varepsilon}} \hat{\mathrm{e}}_{\hat{\mathrm{r}}}\left(\sum_{\tilde{\mathrm{n}}=1}^{\infty}(\tilde{\mathrm{n}}+\mathrm{P})(\tilde{\mathrm{n}}+\mathrm{P}+1)\left(1+\eta^{\prime} \dot{v}\right) a_{\tilde{\mathrm{n}}+\mathrm{P}, \hat{\mathrm{r}}}\right) \leq \sum_{\tilde{\mathrm{r}}=1}^{\grave{\varepsilon}} \hat{\mathrm{e}}_{\hat{\mathrm{r}}} \eta^{\prime} \mathrm{P}(\dot{v}+1)(\mathrm{P}-\dot{\omega}+1)$,

which implies that $G(z)$ is in the class $W \aleph_{P}\left(v^{\prime}, \omega^{\prime}, \eta^{\prime}, \mathrm{T}\right)$. 
In the following, we prove the radius of close- to- convexity.

Theorem (2.6): Let the function $f$ defined by (2) be in the class $W \aleph_{\mathrm{P}}\left(v^{\prime}, \dot{\omega}, \eta \dot{\eta}, \mathrm{T}\right)$.

Then $f$ is close-to-convex of order $(0 \leq \breve{\alpha}<1)$ in $|z|<x_{1}$,

where

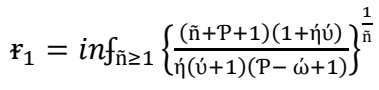

The result is sharp, the external function given (4).

Proof: It is sufficient to show that

$$
\left|\frac{f^{\prime}(z)}{z^{P-1}}-P\right| \leq \text { P for }|z|<x_{1},
$$

where $x_{1}$ is given by (10). Indeed we find from (2) that

$$
\begin{aligned}
\left|\frac{f^{\prime}(z)}{z^{\mathrm{P}-1}}-\mathrm{P}\right| & =\left|\frac{f^{\prime}(z)-P z^{\mathrm{P}-1}}{z^{\mathrm{P}-1}}\right|=\left|\frac{\mathrm{Pz}^{\mathrm{P}-1}-\sum_{\tilde{n}=1}^{\infty}(\tilde{\mathrm{n}}+\mathrm{P}) a_{\tilde{n}+\mathrm{P}^{\tilde{n}+\mathrm{P}-1}-\mathrm{P} z^{\mathrm{P}-1}}}{\mathrm{z}^{\mathrm{P}-1}}\right| \\
& =\left|\frac{-\sum_{\tilde{n}=1}^{\infty}(\tilde{\mathrm{n}}+\mathrm{P}) a_{\tilde{n}+\mathrm{P} z^{\tilde{n}+\mathrm{P}-1}}}{\mathrm{z}^{\mathrm{P}-1}}\right| \leq \sum_{\tilde{\mathrm{n}}=1}^{\infty} \frac{(\tilde{\mathrm{n}}+\mathrm{P}) a_{\tilde{n}+\mathrm{P}}|z|^{\tilde{n}}}{\mathrm{P}} \leq 1,
\end{aligned}
$$

by using Theorem (2.1) and by (12) we get

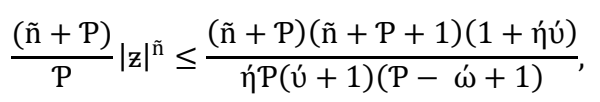

then

$$
|z| \leq\left\{\frac{(\tilde{n}+P+1)(1+\eta \dot{u})}{\tilde{\eta}(\dot{u}+1)(P-\tilde{\omega}+1)}\right\}^{\frac{1}{\tilde{n}}}
$$

The result follows easily from (13).

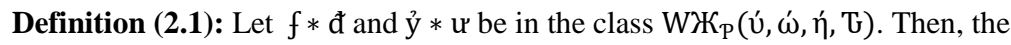

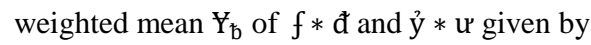

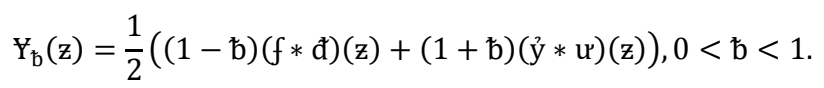

Theorem (2.7): Let $f * đ$ and $y^{3} *$ ư be in the class $W \aleph_{\mathrm{P}}\left(v^{\prime}, \dot{\omega}, \dot{\eta}, \mathrm{T}\right)$. Then, the weighted mean of $f * đ$ and $y^{3} * u$ is also in the class $W \aleph_{\mathrm{P}}\left(v^{\prime}, \omega^{\prime}, \eta^{\prime}, \mathrm{U}\right)$.

Proof: By using Definition (2.1), we get

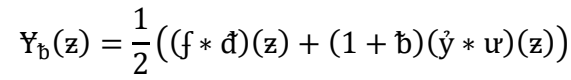

$$
\begin{aligned}
& =\frac{1}{2}\left((1-\mathfrak{b})\left[z^{P}-\sum_{\tilde{n}=1}^{\infty} a_{\tilde{n}+P} 5_{\tilde{n}+P} z^{\tilde{n}+P}\right]+(1+\mathfrak{b})\left[z^{P}-\sum_{\tilde{n}=1}^{\infty} 2_{\tilde{n}+P} \partial_{\tilde{n}+P} z^{\tilde{n}+P}\right]\right) \\
& =z^{P}-\sum_{\tilde{n}=1}^{\infty} \frac{1}{2}\left[(1-\hbar) a_{\tilde{n}+P} 5_{\tilde{n}+P}+(1+\hbar) 2_{\tilde{n}+P} ð_{\tilde{n}+P}\right] z^{\tilde{n}+P} .
\end{aligned}
$$

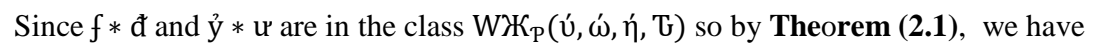




$$
\sum_{\tilde{n}=1}^{\infty}(\tilde{\mathrm{n}}+\mathrm{P})(\tilde{\mathrm{n}}+\mathrm{P}+1)\left(1+\eta^{\prime} u ́\right) a_{\tilde{\mathrm{n}}+\mathrm{P}} 5_{\tilde{\mathrm{n}}+\mathrm{P}} \leq \eta^{\prime} \mathrm{P}(\dot{v}+1)\left(\mathrm{P}-\omega^{\prime}+1\right)
$$

and

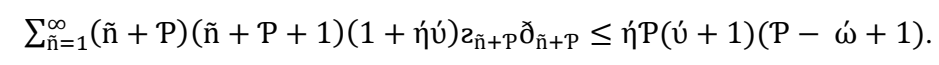

Hence

$$
\begin{aligned}
& \sum_{\tilde{n}=1}^{\infty}(\tilde{n}+P)(\tilde{n}+P+1)\left(1+\eta^{\prime} u\right)\left[\frac{1}{2}(1-\hbar) a_{\tilde{n}+P} 5_{\tilde{n}+P}+\frac{1}{2}(1+\hbar) z_{\tilde{n}+P} ð_{\tilde{n}+P}\right] \\
& =\frac{1}{2}(1-\bar{b}) \sum_{\tilde{n}=1}^{\infty}(\tilde{n}+P)(\tilde{n}+P+1)\left(1+\eta u^{\prime}\right) a_{\tilde{n}+P} 5_{\tilde{n}+P} \\
& =\frac{1}{2}(1+\bar{b}) \sum_{\tilde{n}=1}^{\infty}(\tilde{n}+P)(\tilde{n}+P+1)\left(1+\eta^{\prime} u ́\right) 2_{\tilde{n}+P} \partial_{\tilde{n}+P} \\
& \leq \frac{1}{2}(1-\bar{b}) \eta^{\prime} \mathrm{P}(\dot{v}+1)(\mathrm{P}-\dot{\omega}+1)+\frac{1}{2}(1+\mathrm{b}) \mathfrak{\eta}^{\mathrm{P}} \mathrm{P}(\dot{v}+1)(\mathrm{P}-\dot{\omega}+1) \text {. }
\end{aligned}
$$

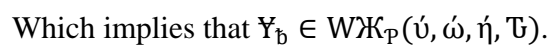

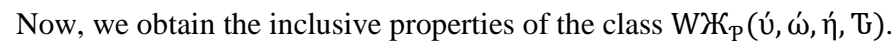

Theorem (2.8): Let $0 \leq \dot{v}<1,0 \leq \dot{\omega}<\mathrm{P}, 0<\dot{\eta}<1$.

Then $W \aleph_{\mathrm{P}}\left(\dot{v}, \dot{\omega}, \eta^{\prime}, \mathrm{T}\right) \subset \mathrm{W} \aleph_{\mathrm{P}}\left(\lambda, \dot{\omega}, \frac{1}{3}, \mathrm{~T}\right)$, where

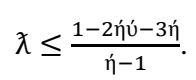

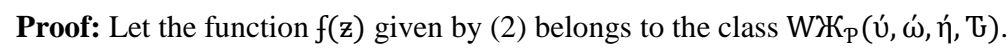

Then in view of Theorem (2.1), we get

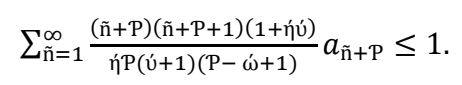

We want to find the value $\chi$ such that

$$
\sum_{\tilde{n}=1}^{\infty} \frac{(\tilde{n}+P)(\tilde{n}+P+1)\left(1+\frac{1}{3} \chi\right)}{\frac{1}{3} P(\lambda+1)(P-\omega+1)} a_{\tilde{n}+P} \leq 1
$$

The inequality (14) would obviously imply (15) if

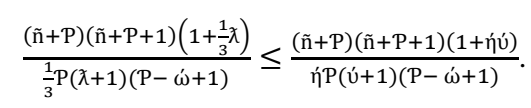

Rewriting the inequality, we get

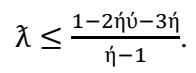

This completes the proof. 


\section{CONFLICT OF INTERESTS}

There are no conflicts of interest.

\section{References}

[1] p. L.Duren, "Univalent Functions", In: Grundlehren der Mathematischen Wissenschaften, Band 259,SpringerVerlag, New York, Berlin, Hidelberg and Tokyo. 1983

[2] A. W. Goodman, " Univalent Functions", Vol. III, Polygonal P, ublishing House, Washington, New Jersey. 1983.

[3] N. Dunford, J.T.Schwarz, " Linear Operator, part I, General Theory", New York- London, Inter Science. 1958.

[4] K.Fan, " Analytic functions of a prOper contraction", Math. Z., Vol.160, pp.275-290. 1978.

[5] K. Fan, " Julia's lemma fOr operators", Math. Ann., Vol. 239, pp. 241- 245. 1979.

[6] Y.Xia0pei, " A subclass of analytic p- valent functions for operator on Hilbert, Space", Math. Japonica, Vol. 40, No.2. pp. 303-308. 1994.

[7] A. K.Wanas," A certain subclass of multivalent analytic functions with negative coefficients for operator on Hilbert space", J. Indones. Math. Soc., Vol. 21, No.2. pp. 77-82. 2015.

[8] W. G. Atshan, R. H. Buti. "Fractional calculus of a class of univalent functions with negative coefficients defined by Hadamard product with Rafid operator". European J. Pure Appl. Math., Vol.4, No.2, pp. 162- 173. 2011.

[9] S.B Joshi, " On a class of analytic functions with negative coefficients for operatOrs on Hilbert Space", J. Appr. Theory and Appl., pp. 107- 112. 1998.

[10] Y.Chrakim, J. S.Lee, S. H.Lee, " A certain subclass of analytic functions with negative coefficients for operators on Hilbert space", Math. Jap0nica, Vol.47, No.1, pp. 155- 124. 1998.

[11] F.Ghanim, M. Darus, " On new subclass of analytic p- valent function with negative coefficients for operators on Hilbert space", Int. Math. Forum, Vol. 3, No.2. pp. 67- 77. 2008.

[12] C.Selvaraj, A.J. Pamela, M.Thirucheran, " On a subclass of multivalent analytic functions with negative coefficients for contraction operators on Hilbert space", Int. J. Contemp. Math. Sci.,Vol.4, No.9. pp. 447- 456. 2009.

\section{بصض الخصائص الهندسية لدالة محدبة متعدة التكافؤ على فضاء هلبرت}

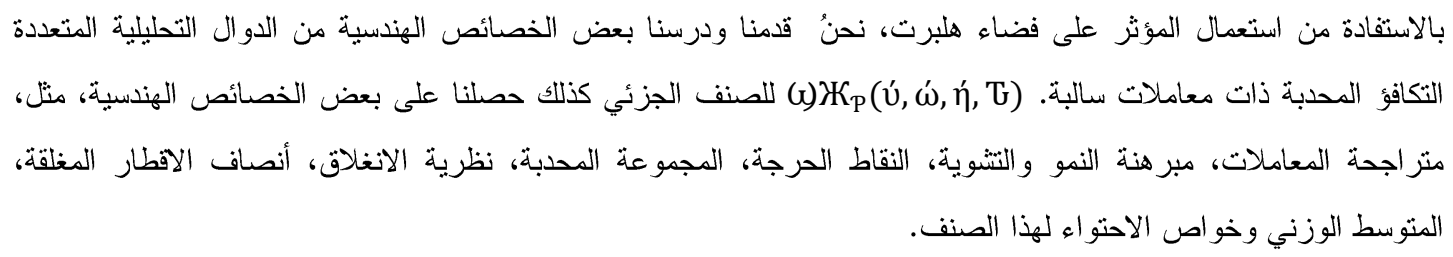

